PROCEEDINGS OF THE

AMERICAN MATHEMATICAL SOCIETY

Volume 137, Number 11, November 2009, Pages 3769-3777

S 0002-9939(09)09941-9

Article electronically published on June 1, 2009

\title{
THE UNIFORM SEPARATION PROPERTY AND BANACH-STONE THEOREMS FOR LATTICE-VALUED LIPSCHITZ FUNCTIONS
}

\author{
A. JIMÉNEZ-VARGAS, A. MORALES CAMPOY, AND MOISÉS VILLEGAS-VALLECILLOS
}

(Communicated by Nigel J. Kalton)

\begin{abstract}
Using the uniform separation property of N. Weaver and the uniform joint property, we present in this paper a Lipschitz version of a BanachStone-type theorem for lattice-valued continuous functions obtained recently by J. X. Chen, Z. L. Chen and N.-C. Wong.
\end{abstract}

\section{INTRODUCTION}

J. Cao, I. Reilly and H. Xiong stated in [4 a lattice-valued version of the classical Banach-Stone theorem on isometries of $\mathcal{C}(X)$-spaces. Since then, several papers have appeared extending this result. We can cite, among others, the generalizations obtained by J. X. Chen, Z. L. Chen and N.-C. Wong [5], Z. Ercan and S. Önal [ 6, 7] and X. Miao, J. Cao and H. Xiong [12.

According to [5, Theorem 3] or [7, Theorem 5], for compact Hausdorff spaces $X$ and $Y$ and Banach lattices $E$ and $F$, if $\mathcal{C}(X, E)$ and $\mathcal{C}(Y, F)$ denote the Banach lattices of continuous $E$-valued and $F$-valued functions defined on $X$ and $Y$, respectively, endowed with the pointwise order and the supremum norm, then every vector lattice isomorphism $T: \mathcal{C}(X, E) \rightarrow \mathcal{C}(Y, F)$ preserving the nowhere vanishing functions in both directions can be written as a weighted composition operator in the form:

$$
T(f)(y)=\widehat{T}(y)(f(\varphi(y)) \quad(f \in \mathcal{C}(X, E), y \in Y),
$$

where $\varphi$ is a homomorphism from $Y$ onto $X$ and $\widehat{T}$ is a continuous map from $Y$ into the space $\mathcal{L}(E, F)$ of all continuous linear operators from $E$ into $F$ equipped with the strong operator topology such that $\widehat{T}(y)$ is a vector lattice isomorphism from $E$ onto $F$ for each $y \in Y$.

Our goal in this paper is to prove this result in the setting of lattice-valued Lipschitz functions. Let us recall that the first results of Banach-Stone type for lattice-valued Lipschitz functions are due to M. I. Garrido and J. A. Jaramillo

Received by the editors February 12, 2009, and, in revised form, February 20, 2009.

2000 Mathematics Subject Classification. Primary 46E40, 46E05.

Key words and phrases. Vector lattice isomorphism, lattice-valued Lipschitz function, BanachStone theorem, uniform separation property.

This research was partially supported by Junta de Andalucía grants FQM-1438 and FQM-3737, and MCYT projects MTM2006-4837 and MTM2007-65959.

The third author was supported in part by Beca Plan Propio Universidad de Almería.

(C)2009 American Mathematical Society 
[8. Theorem 3.10] and N. Weaver [13, Main theorem, Part (d)], and they concern real-valued Lipschitz functions. The first two authors also tackled the matter for vector lattices of real-valued locally Lipschitz functions [8, Theorem 3.16] and real-valued Lipschitz functions in the small [9, Theorem 2], and N. Weaver tackled normal lattices of complex-valued Lipschitz functions vanishing at some fixed point [14, Corollary]. Recently, we have given in [11 the general form of order isomorphisms between spaces of real or complex-valued little Lipschitz functions on compact Hölder metric spaces. It is worthwhile noting also that versions not necessarily linear of Banach-Stone-type theorems for different lattices of real-valued Lipschitz functions have been stated very recently by F. and J. Cabello Sánchez in 3. See 2 for related results.

Let $(X, d)$ be a metric space and let $E$ be a nonzero Banach lattice. We will denote by $\operatorname{Lip}(X, E)$ the complete normed vector lattice of all bounded Lipschitz functions from $X$ into $E$, equipped with the pointwise order and the norm $\|f\|_{d}=$ $\max \left\{L_{d}(f),\|f\|_{\infty}\right\}$ where $L_{d}(f)$ is the Lipschitz number of $f$.

We want to formulate in this paper Banach-Stone-type theorems for vector lattice isomorphisms defined between different vector sublattices of $\operatorname{Lip}(X, E)$. To this end, we will extend here the uniform separation property by N. Weaver [15, 16] and introduce the uniform joint property (see Definitions 3.1 and 3.2). Moreover, we will show that the class of vector sublattices of $\operatorname{Lip}(X, E)$ satisfying these properties is sufficiently large. In this way, under the condition of compactness on $X$ and $Y$, we will prove that if $\mathrm{A}(X, E)$ and $\mathrm{A}(Y, F)$ are vector sublattices of $\operatorname{Lip}(X, E)$ and $\operatorname{Lip}(Y, F)$, respectively, that separate and join points uniformly, then every vector lattice isomorphism $T$ from $\operatorname{Lip}(X, E)$ onto $\operatorname{Lip}(Y, F)$ that preserves the nowhere vanishing functions in both directions can be expressed in the form (1.1). Of course,

the functions $\varphi$ and $\widehat{T}$ that will appear in this representation of $T$ will be Lipschitz.

The content of the paper is as follows. Section 2 is devoted to generalities on vector lattices and Lipschitz functions, Section 3 focuses on the uniform separation and joint properties and, finally, Section 4 contains our main result and an application to the real-valued case.

\section{LATticE-VAlued LiPschitz FUnCtions}

We first recall some concepts on vector lattices. A vector lattice $E$ is an ordered vector space in which $x \vee y$ exists for every $x, y \in E$. For each $x \in E,|x|=x \vee(-x)$ is the absolute value of $x$. A Banach lattice $E$ is a vector lattice equipped with a complete norm $\|\cdot\|$ that satisfies the so-called Riesz law: for any $x, y \in E,|x| \leq|y|$ implies $\|x\| \leq\|y\|$. A linear map between vector lattices $T: E \rightarrow F$ is said to be a vector lattice homomorphism if $T(x \vee y)=T(x) \vee T(y)$ for every $x, y \in E$. A bijective vector lattice homomorphism is called a vector lattice isomorphism. Clearly, the inverse of a vector lattice isomorphism is a vector lattice isomorphism. A linear map between vector lattices $T: E \rightarrow F$ is positive if $T(x) \geq 0$ whenever $x \in E$ and $x \geq 0$. Notice that if $T: E \rightarrow F$ is a vector lattice homomorphism, then $T(|x|)=|T(x)|$ for all $x \in E$, and therefore $T$ is positive.

We next present some vector lattices of Lipschitz functions. Let $(X, d)$ be a metric space and let $E$ be a nonzero Banach space. A map $f: X \rightarrow E$ is said to be Lipschitz if

$$
L_{d}(f)=\sup \{\|f(x)-f(y)\| / d(x, y): x, y \in X, x \neq y\}<\infty .
$$


The Lipschitz space $\operatorname{Lip}(X, E)$ is the Banach space of all bounded Lipschitz functions $f: X \rightarrow E$ with the Lipschitz norm

$$
\|f\|_{d}=\max \left\{L_{d}(f),\|f\|_{\infty}\right\}
$$

where

$$
\|f\|_{\infty}=\sup \{\|f(x)\|: x \in X\} .
$$

The little Lipschitz space $\operatorname{lip}(X, E)$ is the norm-closed subspace of $\operatorname{Lip}(X, E)$ formed by all those functions $f$ such that $\lim _{d(x, y) \rightarrow 0}\|f(x)-f(y)\| / d(x, y)=0$; that is,

$$
\forall \epsilon>0, \exists \delta>0: x, y \in X, 0<d(x, y)<\delta \Rightarrow\|f(x)-f(y)\| / d(x, y)<\epsilon .
$$

Hölder functions are of special interest in relation to little Lipschitz functions. Given a metric space $(X, d)$ and a real number $0<\alpha<1$, the map $d^{\alpha}: X \times X \rightarrow \mathbb{R}_{0}^{+}$ defined by $d^{\alpha}(x, y)=d(x, y)^{\alpha}$ is a metric on $X$. Following [17, Definition 1.1.2], we will denote by $X^{\alpha}$ the metric space $\left(X, d^{\alpha}\right)$. We say that $X^{\alpha}$ is a Hölder metric space, and the elements of $\operatorname{Lip}\left(X^{\alpha}\right)$ are called Hölder functions.

If, in addition, $E$ is a Banach lattice, then $\operatorname{Lip}(X, E)$ is a vector lattice with the pointwise order:

$$
f \leq g \Leftrightarrow f(x) \leq g(x), \forall x \in X
$$

However, $\operatorname{Lip}(X, E)$ is not, in general, a Banach lattice since the norm $\|\cdot\|_{d}$ does not satisfy the Riesz law. Moreover, $\operatorname{lip}(X, E)$ is a vector sublattice of $\operatorname{Lip}(X, E)$. In the case $E=\mathbb{R}$, we will delete the letter $E$ and write $\operatorname{simply} \operatorname{Lip}(X)$ and $\operatorname{lip}(X)$.

A pointed metric space is a metric space $X$ with a distinguished element $e_{X} \in X$ called the base point. Given a pointed metric space $X$ and a nonzero Banach lattice $E, \operatorname{Lip}_{0}(X, E)$ denotes the norm-closed vector sublattice of $\operatorname{Lip}(X, E)$ formed by all functions that vanish at $e_{X}$.

Let us recall that a map between metric spaces $\varphi: Y \rightarrow X$ is said to be $b i$ Lipschitz if it is bijective and both $\varphi$ and $\varphi^{-1}$ are Lipschitz. Given a metric space $X$, we will denote its diameter by $\Delta(X)$ and the function constantly 1 on $X$ by $1_{X}$. For a nonzero Banach space $E, S_{E}$ will stand for the unit sphere of $E$.

Let $X$ and $Y$ be metric spaces, $E$ and $F$ nonzero Banach spaces and $A(X, E)$ and $A(Y, F)$ nonempty subsets of $\operatorname{Lip}(X, E)$ and $\operatorname{Lip}(Y, F)$, respectively. We say that a map $f: X \rightarrow E$ is nowhere vanishing if $f(x) \neq 0$ for all $x \in X$, and a map $T$ : $A(X, E) \rightarrow A(Y, F)$ preserves the nowhere vanishing functions if $T(f)$ is nowhere vanishing whenever $f$ is. If $T$ is bijective, it is said that $T$ preserves the nowhere vanishing functions in both directions if $T$ and $T^{-1}$ preserve the nowhere vanishing functions. If $E$ is a nonzero Banach lattice, a map $f: X \rightarrow E$ is everywhere positive if $f(x)>0$ for all $x \in X$.

For a comprehensive study of positive operators between Banach lattices, we refer the reader to the monograph [1] by C. D. Aliprantis and O. Burkinshaw. In particular, the lattice structure of spaces of Lipschitz functions has been studied by N. Weaver in [17, Chapter 5]. Moreover, this book contains complete information on Lipschitz functions.

\section{THE UNIFORM SEPARATION AND JOINT PROPERTIES}

Let $X$ be a compact metric space. N. Weaver introduced in [15] (see also [16, Theorem 1]) a kind of uniform separation condition of the points of $X$ by means of functions in $\operatorname{lip}(X)$. We extend here this property for any vector subspace $A(X, E)$ of $\operatorname{Lip}(X, E)$ as follows. If $E=\mathbb{R}$, we will write simply $A(X)$. 
Definition 3.1. Let $(X, d)$ be a compact metric space and let $E$ be a nonzero Banach space. We say that a vector subspace $A(X, E)$ of $\operatorname{Lip}(X, E)$ separates points uniformly if there exists a constant $a>1$ such that for every $x, y \in X$ and $e \in S_{E}$, there is a function $h \in A(X, E)$, depending on $x, y$ and $e$, with $\|h\|_{d} \leq a$ such that $h(x)=d(x, y) e$ and $h(y)=0$.

For any $f \in \operatorname{Lip}(X)$ and $e \in E$, denote by $f \cdot e$ the function of $X$ to $E$ defined by setting $(f \cdot e)(x)=f(x) e$ for all $x \in X$. Notice that $f \cdot e \in \operatorname{Lip}(X, E)$ and $\|f \cdot e\|_{d}=\|f\|_{d}\|e\|$.

Let $A$ be a vector subspace of $\operatorname{Lip}(X)$. It is obvious that $\operatorname{Lip}(X, E), \operatorname{lip}(X, E)$ and $\operatorname{Lip}_{0}(X, E)$ contain the set $\left\{f \cdot e: f \in A, e \in S_{E}\right\}$ with $A$ equal to $\operatorname{Lip}(X), \operatorname{lip}(X)$ and $\operatorname{Lip}_{0}(X)$, respectively. If $A(X, E)$ is a vector subspace of $\operatorname{Lip}(X, E)$ which contains the set $\left\{f \cdot e: f \in A, e \in S_{E}\right\}$, it is clear that $A(X, E)$ has the uniform separation property if $A$ does so. Next we present some vector subspaces of $\operatorname{Lip}(X)$ satisfying this property.

Let us recall that $\operatorname{Lip}(X)$ separates points uniformly with $a=\max \{1, \Delta(X)\}$. Notice that for every $x, y \in X$, the function $h \in \operatorname{Lip}(X)$ defined by $h(z)=d(z, y)$ and its negative $-h$ satisfies the required conditions. As well, $\operatorname{Lip}_{0}(X)$ separates points uniformly. Indeed, given $x, y \in X$ with $x \neq y$, assume $d\left(y, e_{X}\right) \leq d\left(x, e_{X}\right)$ and then $d(x, y) \leq 2 d\left(x, e_{X}\right)$. Take $\delta=\min \left\{d\left(x, e_{X}\right), d(x, y)\right\}$ and define $h(z)=$ $d(x, y) \max \{1-d(z, x) / \delta, 0\}$ on $X$. An easy verification shows that $h \in \operatorname{Lip}_{0}(X)$ with $\|h\|_{d} \leq a=\max \{2, \Delta(X)\}, h(x)=d(x, y)$ and $h(y)=0$.

On the other hand, $\operatorname{lip}(X)$ in general does not have the uniform separation property. For instance, if $X=[0,1]$ with the usual metric, $\operatorname{lip}(X)$ consists only of constant functions. More generally, the same conclusion can be drawn for a connected and complete Riemannian manifold $X$ [17, Example 3.1.5]. Nevertheless, if $X$ is uniformly discrete, that is, if there is a number $\delta>0$ such that $d(x, y) \geq \delta$ for all $x, y \in X$ with $x \neq y$, then $\operatorname{lip}(X)=\operatorname{Lip}(X)$ and thus $\operatorname{lip}(X)$ has the uniform separation property.

Other, deeper examples of spaces of little Lipschitz functions that separate points uniformly are: (a) $\operatorname{lip}(K)$, where $K$ is the middle-thirds Cantor set with the metric inherited from $[0,1]$, with constant $a=3$; and (b) $\operatorname{lip}\left(X^{\alpha}\right)$, where $0<\alpha<1$ and $X$ is a compact metric space, with $a=2^{(1-\alpha) / \alpha} \max \{1, \Delta(X)\}$. See [17, Proposition $3.2 .2]$.

We now introduce the uniform joint property.

Definition 3.2. Let $(X, d)$ be a compact metric space and let $E$ be a nonzero Banach space. We say that a vector subspace $A(X, E)$ of $\operatorname{Lip}(X, E)$ joins points uniformly if there exists a constant $b>1$ such that for every $x, y \in X$ and $e \in S_{E}$, there is a function $k \in A(X, E)$, depending on $x, y$ and $e$, with $\|k\|_{d} \leq b$ such that $k(x)=k(y)=e$.

Let $A$ be a vector subspace of $\operatorname{Lip}(X)$. If $A(X, E)$ is a vector subspace of $\operatorname{Lip}(X, E)$ containing the set $\left\{f \cdot e: f \in A, e \in S_{E}\right\}$, it is evident that $A(X, E)$ joins points uniformly if $A$ does so. Notice that $A$ joins points uniformly if it contains the constant functions. Hence $\operatorname{Lip}(X, E)$ and $\operatorname{lip}(X, E)$ have the uniform joint property. On the other hand, it is clear that $\operatorname{Lip}_{0}(X, E)$ does not join points.

The space $\mathcal{C}^{1}([0,1])$ of all continuously differentiable functions from $[0,1]$ to $\mathbb{R}$ is a normed-closed subspace of $\operatorname{Lip}([0,1])$ that separates points uniformly and contains the constant functions, but it is not lattice-ordered. In fact, we are interested 
only in vector sublattices of Lipschitz functions on compact metric spaces, and all the examples of such sublattices that join points uniformly seen so far contain the constant functions. Therefore we finish this section with an example of a vector sublattice of Lipschitz functions on a compact metric space that separates and joins points uniformly, but it does not contain the constant functions.

Example 3.3. Let $X=[0,1]$ be equipped with the usual metric and define $f, g$ : $X \rightarrow \mathbb{R}$ by $f(t)=-(t-2)$ and $g(t)=t^{2}-4$. Clearly, $f, g \in \operatorname{Lip}(X)$ with $\|f\|_{d}=2$ and $\|g\|_{d}=4$. Take the real vector space $B=\operatorname{lin}(\{f, g\})$ and let $A$ be the vector sublattice of $\operatorname{Lip}(X)$ generated by $B$. Let us recall that $A$ is equal to ${ }^{\vee}(\wedge B)$ by [10, 2.2.11], where ${ }^{\wedge} B$ is the set of all finite infima from $B$ and ${ }^{\vee}\left({ }^{\wedge} B\right)$ is the set of all finite suprema from ${ }^{\wedge} B$.

We first show that $A$ separates and joins points uniformly. Let $x, y \in X, x \neq y$ and $c, d \in \mathbb{R}$ be given. Consider the function $\alpha f+\beta g$ defined on $X$, where $\alpha$ and $\beta$ are the solutions of the system

$$
\begin{aligned}
& \alpha f(x)+\beta g(x)=c, \\
& \alpha f(y)+\beta g(y)=d .
\end{aligned}
$$

These solutions exist because the determinant of coefficients is $(x-2)(y-2)(x-y) \neq$ 0 . In particular, if $c=|x-y|$ and $d=0$, we have $\alpha=(y+2)|x-y| /(x-2)(x-y)$ and $\beta=|x-y| /(x-2)(x-y)$, and the function $h=\alpha f+\beta g \in B$ satisfies $h(x)=|x-y|, h(y)=0$ and $\|h\|_{d} \leq 10$. On the other hand, for $c=d=1$, we get $\alpha=-(x+y) /(x-2)(y-2)$ and $\beta=-1 /(x-2)(y-2)$, and the function $k=\alpha f+\beta g \in B$ fulfills that $k(x)=k(y)=1$ and $\|k\|_{d} \leq 8$. If $c=d=-1$, take $-k$.

It remains to prove that $A$ does not contain the constant functions. First notice that if $f_{1} \in B$ is constant on an open set $G \subseteq X$, then $f_{1}=0$. Indeed, if $f_{1}=$ $\alpha f+\beta g$ is constant on $G$, then $\alpha f^{\prime}+\beta g^{\prime}=0$ on $G$; that is, $-\alpha+2 \beta t=0$ for all $t \in G$, which implies $\alpha=\beta=0$ and thus $f_{1}=0$. On the other hand, it is easy to show that if $f_{1}, f_{2} \in \operatorname{Lip}(X), \alpha \in \mathbb{R}$ and $f_{1} \vee f_{2}=\alpha$ on an open $G \subseteq X$, then either $f_{1}=\alpha$ on an open $G_{1} \subseteq X$ with $G_{1} \subseteq G$ or $f_{2}=\alpha$ on an open $G_{2} \subseteq X$ with $G_{2} \subseteq G$. The same assertion holds for $f_{1} \wedge f_{2}$ instead of $f_{1} \vee f_{2}$.

We next prove by induction on $n \in \mathbb{N}$ that if $f_{1}, \ldots, f_{n} \in B$ and $f_{1} \wedge \ldots \wedge f_{n}$ is constant on an open $G \subseteq X$, then $f_{1} \wedge \ldots \wedge f_{n}=0$ on $G$. For $n=1$, the assertion has been proved. Let us suppose that it is true for $n$, and we prove it for $n+1$. Assume that there are $\alpha \in \mathbb{R}$ and an open $G \subseteq X$ such that $f_{1} \wedge \ldots \wedge f_{n+1}=\alpha$ on $G$. Then there exists an open $G_{1} \subseteq X$ with $G_{1} \subseteq G$ such that $f_{1} \wedge \ldots \wedge f_{n}=\alpha$ on $G_{1}$ or there exists an open $G_{2} \subseteq X$ with $G_{2} \subseteq G$ such that $f_{n+1}=\alpha$ on $G_{2}$. It follows that $f_{1} \wedge \ldots \wedge f_{n}=0$ on $G_{1}$ or $f_{n+1}=0$ on $G_{2}$. Since $f_{1} \wedge \ldots \wedge f_{n+1}=\alpha$ on $G$, we conclude in both cases that $f_{1} \wedge \ldots \wedge f_{n+1}=0$ on $G$. In this way we have proved that if $r \in \wedge^{\wedge} B$ is constant on an open $G \subseteq X$, then $r=0$ on $G$. Similarly, it is proved that if $s \in{ }^{\vee}\left({ }^{\wedge} B\right)$ is constant on an open $G \subseteq X$, then $s=0$ on $G$. Therefore the unique constant function contained in $A$ is 0 .

\section{BAnaCh-Stone theOREMS}

We are ready to state the main result of the paper. For Banach spaces $E$ and $F$, we will consider that the space $\mathcal{L}(E, F)$ of all continuous linear operators from $E$ into $F$ is equipped with the metric inherited from the operator canonical norm. 
Theorem 4.1. Let $T: A(X, E) \rightarrow A(Y, F)$ be a vector lattice isomorphism that preserves the nowhere vanishing functions in both directions, where $X$ and $Y$ are compact metric spaces, $E$ and $F$ are nonzero Banach lattices and $A(X, E)$ and $A(Y, F)$ are vector sublattices of $\operatorname{Lip}(X, E)$ and $\operatorname{Lip}(Y, F)$, respectively, that separate and join points uniformly. Then there exist a bi-Lipschitz map $\varphi: Y \rightarrow X$ and a Lipschitz map $\widehat{T}: Y \rightarrow \mathcal{L}(E, F)$ where $\widehat{T}(y): E \rightarrow F$ is a vector lattice isomorphism for each $y \in Y$ such that $T(f)(y)=\widehat{T}(y)(f(\varphi(y))$ for every $f \in A(X, E)$ and all $y \in Y$.

Proof. For any $x \in X$ and $y \in Y$, define the sets

$$
\begin{aligned}
M_{x} & =\{f \in A(X, E): f(x)=0\}, \\
N_{y} & =\{g \in A(Y, F): g(y)=0\} .
\end{aligned}
$$

We first see that if $x_{1}, x_{2} \in X$ and $M_{x_{1}} \subseteq M_{x_{2}}$, then $x_{1}=x_{2}$. Indeed, assume that $x_{1} \neq x_{2}$. Since $A(X, E)$ separates points, then, for some $e \in S_{E}$, there exists $h \in A(X, E)$ for which $h\left(x_{1}\right)=0$ and $h\left(x_{2}\right)=d\left(x_{1}, x_{2}\right) e$, and thus $h \in M_{x_{1}} \backslash M_{x_{2}}$. In the same manner we can prove that $y_{1}=y_{2}$ provided $y_{1}, y_{2} \in Y$ and $N_{y_{1}} \subseteq N_{y_{2}}$.

The key point of the proof is the fact that for every $y \in Y$, there exists a unique $x \in X$ such that $T\left(M_{x}\right)=N_{y}$. In order to prove this, fix $y \in Y$. We first claim that $T^{-1}\left(N_{y}\right) \subseteq M_{x}$ for some $x \in X$. For each $g \in N_{y}$, denote by $\mathrm{Z}\left(T^{-1}(g)\right)$ the set $\left\{x \in X: T^{-1}(g)(x)=0\right\}$. Notice that $\mathrm{Z}\left(T^{-1}(g)\right)$ is nonempty for every $g \in N_{y}$ since $T$ preserves the nowhere vanishing functions. The claim will follow if we prove that $\bigcap_{g \in N_{y}} \mathrm{Z}\left(T^{-1}(g)\right)$ is nonempty. To see this, we only need to check that the family of closed subsets of the compact space $X$, $\left\{\mathrm{Z}\left(T^{-1}(g)\right): g \in N_{y}\right\}$, has the finite intersection property. Pick $g_{1}, \ldots, g_{n} \in N_{y}$ and take $g=\bigvee_{k=1}^{n}\left|g_{k}\right|$. It is clear that $g \in N_{y}$ and $T^{-1}(g)=\bigvee_{k=1}^{n}\left|T^{-1}\left(g_{k}\right)\right|$. An easy verification gives $\mathrm{Z}\left(T^{-1}(g)\right)=\bigcap_{k=1}^{n} \mathrm{Z}\left(T^{-1}\left(g_{k}\right)\right)$. Since $\mathrm{Z}\left(T^{-1}(g)\right)$ is nonempty, so is $\bigcap_{k=1}^{n} \mathrm{Z}\left(T^{-1}\left(g_{k}\right)\right)$ as we desired. This proves our claim, and therefore there is an $x \in X$ such that $T^{-1}\left(N_{y}\right) \subseteq M_{x}$. Since $T^{-1}$ has the same properties as $T$, we can likewise find a point $y^{\prime} \in Y$ such that $T\left(M_{x}\right) \subseteq N_{y^{\prime}}$. Then $N_{y}=T\left(T^{-1}\left(N_{y}\right)\right) \subseteq T\left(M_{x}\right) \subseteq N_{y^{\prime}}$. This yields $y=y^{\prime}$ and thus $T\left(M_{x}\right)=N_{y}$.

To show that this $x$ is unique, assume that $T\left(M_{x^{\prime}}\right)=N_{y}$ for some $x^{\prime} \in X$. Then $M_{x}=T^{-1}\left(N_{y}\right)=M_{x^{\prime}}$ and so $x=x^{\prime}$.

In view of what has been proven above, we can define, with a slight abuse of notation, a map $\varphi: Y \rightarrow X$ by

$$
T\left(M_{\varphi(y)}\right)=N_{y} \quad(y \in Y) .
$$

Since $T^{-1}$ has a similar property as $T$, we can apply the argument above to $T^{-1}$ and obtain a map $\phi: X \rightarrow Y$ such that

$$
T^{-1}\left(N_{\phi(x)}\right)=M_{x} \quad(x \in X) .
$$

It is clear that, for every $y \in Y, N_{\phi(\varphi(y))}=T\left(T^{-1}\left(N_{\phi(\varphi(y))}\right)\right)=T\left(M_{\varphi(y)}\right)=N_{y}$, and hence $\phi(\varphi(y))=y$. Thus $\phi \circ \varphi$ is the identity map on $Y$. In a similar way, it is proved that $\varphi \circ \phi$ is the identity map on $X$. These facts imply that $\varphi$ is bijective and $\varphi^{-1}=\phi$.

We now use the map $\varphi$ to obtain the functional representation of $T$ that appears in the statement of the theorem. First notice that for each $y \in Y$, we have $E=$ $\{f(\varphi(y)): f \in A(X, E)\}$ since $A(X, E)$ joins points. For every $y \in Y$, define the 
map $\widehat{T}(y): E \rightarrow F$ by the formula

$$
\widehat{T}(y)(f(\varphi(y)))=T(f)(y) \quad(f \in A(X, E)) .
$$

According to (4.1), $\widehat{T}(y)$ is a well-defined linear injective map on $E$. On the other hand, given $u \in F$, we can take a function $g \in A(Y, F)$ for which $g(y)=u$ since $A(Y, F)$ joins points, and the surjectivity of $T$ gives us $T(f)=g$ for some $f \in$ $A(X, E)$. Then $\widehat{T}(y)(f(\varphi(y)))=T(f)(y)=g(y)=u$, and this proves that $\widehat{T}(y)$ is surjective. Finally, for any $f_{1}, f_{2} \in A(X, E)$, we have

$$
\begin{aligned}
\widehat{T}(y)\left(f_{1}(\varphi(y)) \vee f_{2}(\varphi(y))\right) & =\widehat{T}(y)\left(\left(f_{1} \vee f_{2}\right)(\varphi(y))\right) \\
& =T\left(f_{1} \vee f_{2}\right)(y) \\
& =T\left(f_{1}\right)(y) \vee T\left(f_{2}\right)(y) \\
& =\widehat{T}(y)\left(f_{1}(\varphi(y))\right) \vee \widehat{T}(y)\left(f_{2}(\varphi(y))\right),
\end{aligned}
$$

since $T$ is a vector lattice homomorphism. We have proved thus that $\widehat{T}(y)$ is a vector lattice isomorphism.

Let us recall that every positive linear map and, in particular, every vector lattice homomorphism between Banach lattices is norm-continuous (see, for example, 1, Theorem 4.3]). As a consequence, $\widehat{T}(y)$ is norm-continuous for every $y \in Y$.

Moreover, an analysis of the proof of this theorem reveals that the assumption that the Banach lattices satisfy the Riesz law is unimportant. Therefore $T: A(X, E) \rightarrow A(Y, F)$ is automatically continuous for the respective Lipschitz norms.

In order to prove that $\widehat{T}$ is a Lipschitz map from $Y$ into $\mathcal{L}(E, F)$, fix $y, z \in Y$. By assumption, there exists a constant $b>1$ satisfying that for every $e \in S_{E}$, there is a function $k \in A(X, E)$, depending on $y, z$ and $e$, with $\|k\|_{d} \leq b$ such that $k(\varphi(y))=k(\varphi(z))=e$. Then we have

$$
\begin{aligned}
\|(\widehat{T}(y)-\widehat{T}(z))(e)\| & =\|\widehat{T}(y)(k(\varphi(y)))-\widehat{T}(z)(k(\varphi(z)))\| \\
& =\|T(k)(y)-T(k)(z)\| \\
& \leq L_{d}(T(k)) d(y, z) \\
& \leq b\|T\| d(y, z),
\end{aligned}
$$

and we conclude that $\|\widehat{T}(y)-\widehat{T}(z)\| \leq b\|T\| d(y, z)$.

We now show that $\varphi$ is Lipschitz. Let $a>1$ be the constant involved in the definition of the uniform separation property of $A(X, E)$. Fix $e \in S_{E}$. For every $y, z \in Y$, there exists $h \in A(X, E)$, depending on $y, z$ and $e$, with $\|h\|_{d} \leq a$ such that $h(\varphi(y))=d(\varphi(y), \varphi(z)) e$ and $h(\varphi(z))=0$. Since $T$ is linear and continuous, we have $\|T(h)\|_{d} \leq a\|T\|$. It follows that

$$
\|T(h)(y)-T(h)(z)\| \leq a\|T\| d(y, z) .
$$

Applying the functional representation of $T$ gives

$$
\begin{aligned}
& T(h)(y)=\widehat{T}(y)(h(\varphi(y)))=d(\varphi(y), \varphi(z)) \widehat{T}(y)(e), \\
& T(h)(z)=\widehat{T}(z)(h(\varphi(z)))=0 .
\end{aligned}
$$

Substituting this in the inequality above, we obtain

$$
d(\varphi(y), \varphi(z))\|\widehat{T}(y)(e)\| \leq a\|T\| d(y, z) .
$$


Notice that $\inf _{y \in Y}\|\widehat{T}(y)(e)\|=\left\|\widehat{T}\left(y_{0}\right)(e)\right\|:=c>0$ for some $y_{0} \in Y$, since the real function $y \mapsto\|\widehat{T}(y)(e)\|$ defined on the compact $Y$ is continuous by (4.3), and $\widehat{T}\left(y_{0}\right)$ is linear and injective. Then $d(\varphi(y), \varphi(z)) \leq(a / c)\|T\| d(y, z)$, and so $\varphi$ is Lipschitz.

Since $T^{-1}$ has the same properties as $T$, from what has already been proved we deduce that the map $\phi: X \rightarrow Y$ defined in (4.2) is Lipschitz, but $\phi=\varphi^{-1}$ as was shown. Hence $\varphi^{-1}$ is Lipschitz, and this completes the proof of the theorem.

We finish the paper with an application for real-valued Lipschitz functions. This generalizes [11, Theorem 2.4].

Corollary 4.2. Let $T: A(X) \rightarrow A(Y)$ be a vector lattice isomorphism, where $X$ and $Y$ are compact metric spaces and $A(X)$ and $A(Y)$ are vector sublattices of $\operatorname{Lip}(X)$ and $\operatorname{Lip}(Y)$, respectively, that separate points uniformly and contain the constant functions. Then $T(f)=\tau \cdot(f \circ \varphi)$ for every $f \in A(X)$, where $\varphi: Y \rightarrow X$ is a bi-Lipschitz map and $\tau=T\left(1_{X}\right) \in A(Y)$ is an everywhere positive function.

Proof. Notice first that $T$ preserves the nowhere vanishing functions in both directions. Indeed, take $v \in A(X)$ such that $T(v)=1_{Y}$. By the compactness of $X, v \leq \alpha 1_{X}$ for some $\alpha \in \mathbb{R}^{+}$. Hence $1_{Y}=T(v) \leq \alpha T\left(1_{X}\right)$, and thus $T\left(1_{X}\right)$ is nowhere vanishing. Now, let $f \in A(X)$ be nowhere vanishing. Likewise, $|f| \geq \beta 1_{X}$ for some $\beta \in \mathbb{R}^{+}$; then $|T(f)|=T(|f|) \geq \beta T\left(1_{X}\right)$ and so $T(f)$ is nowhere vanishing. Hence $T$ preserves the nowhere vanishing functions. The same reasoning applies to $T^{-1}$.

According to Theorem 4.1 there are a bi-Lipschitz map $\varphi: Y \rightarrow X$ and a vector lattice isomorphism $\widehat{T}(y)$ from $\mathbb{R}$ onto itself for each $y \in Y$ such that $T(f)(y)=$ $\widehat{T}(y)(f(\varphi(y))$ for every $f \in A(X)$ and all $y \in Y$. Hence $T(f)(y)=f(\varphi(y)) \widehat{T}(y)(1)$, and thus $T(f)(y)=T\left(1_{X}\right)(y) f(\varphi(y))$.

\section{REFERENCES}

1. C. D. Aliprantis and O. Burkinshaw, Positive Operators, Pure and Applied Mathematics, vol. 119, Academic Press, New York, 1985. MR809372 (87h:47086)

2. F. Cabello Sánchez, J. Cabello Sánchez, Z. Ercan and S. Önal, Memorandum on multiplicative bijections and order, available at http://kolmogorov.unex.es/ fcabello.

3. F. Cabello Sánchez and J. Cabello Sánchez, Nonlinear isomorphisms of lattices of Lipschitz functions, available at http://kolmogorov.unex.es/ ${ }^{\sim}$ cabello.

4. J. Cao, I. Reilly and H. Xiong, A lattice-valued Banach-Stone theorem, Acta Math. Hungar. 98 (2003), 103-110. MR.1958470 (2003m:46028)

5. J. X. Chen, Z. L. Chen and N.-G. Wong, A Banach-Stone theorem for Riesz isomorphisms of Banach lattices, Proc. Amer. Math. Soc. 136 (2008), 3869-3874. MR2425726

6. Z. Ercan and S. Önal, Banach-Stone theorem for Banach lattice valued continuous functions, Proc. Amer. Math. Soc. 135 (2007), 2827-2829. MR2317958(2008a:46038)

7. Z. Ercan and S. Önal, The Banach-Stone theorem revisited, Topology Appl. 155 (2008), 1800-1803. MR2445303

8. M. I. Garrido and J. A. Jaramillo, Homomorphisms on function lattices, Monatsh. Math. 141 (2004), 127-146. MR2037989(2004k:46034)

9. M. I. Garrido and J. A. Jaramillo, Lipschitz-type functions on metric spaces, J. Math. Anal. Appl. 340 (2008), 282-290. MR2376153(2008m:46063)

10. G. J. O. Jameson, Ordered Linear Spaces, Lecture Notes in Math., vol. 141, Springer-Verlag, Berlin, 1970. MR0438077 (55:10996)

11. A. Jiménez-Vargas and Moisés Villegas-Vallecillos, Order isomorphisms of little Lipschitz algebras, Houston J. Math. 34 (2008), 1185-1195. MR2465374 
12. X. Miao, J. Cao and H. Xiong, Banach-Stone theorems and Riesz algebras, J. Math. Anal. Appl. 313 (2006), 177-183. MR2178729 (2006m:46030)

13. N. Weaver, Lattices of Lipschitz functions, Pacific. J. Math. 164 (1994), 179-193. MR.1267506 (95b:46031)

14. N. Weaver, Order completeness in Lipschitz algebras, J. Funct. Anal. 130 (1995), 118-130. MR 1331979 (96f:46048)

15. N. Weaver, Subalgebras of little Lipschitz algebras, Pacific. J. Math. 173 (1996), 283-293. MR:1387803 (97f:46083)

16. N. Weaver, Quotients of little Lipschitz algebras, Proc. Amer. Math. 125 (1997), 2643-2648. MR.1402889 (97j:46021)

17. N. Weaver, Lipschitz algebras, World Scientific Publishing Co., River Edge, NJ, 1999. MR 1832645 (2002g:46002)

Departamento de Álgebra y Análisis Matemático, Universidad de Almería, 04120 Almería, Spain

E-mail address: ajimenez@ual.es

Departamento de Álgebra y Análisis Matemático, Universidad de Almería, 04120 Almería, SPAin

E-mail address: amorales@ual.es

Departamento de Álgebra y Análisis Matemático, Universidad de Almería, 04120 Almería, Spain

E-mail address: mvv042@alboran.ual.es 